\title{
Improves Network Capacity using Topology Control in Wireless Network
}

\author{
J.Ramesh Babu ${ }^{1}$, P.Praveen Kumar ${ }^{2}$ \\ Assistant Professor, B.V.Raju Institute of Technology, Narasapur, JNTU Hyderabad, India ${ }^{1}$ \\ Pursuing M.Tech, B.V.Raju Institute of Technology, Narasapur, JNTU Hyderabad, India ${ }^{2}$
}

\begin{abstract}
Cooperative communication has received tremendous interest for wireless networks. Most existing works on cooperative communications are focused on link-level physical layer issues. Consequently, the impacts of cooperative communications on network-level upper layer issues, such as topology control, routing and network capacity, are largely ignored. In this article, we propose a Capacity-Optimized Cooperative (COCO) topology control scheme to improve the network capacity in MANETs by jointly considering both upper layer network capacity and physical layer cooperative communications. Through simulations, we show that physical layer cooperative communications have significant impacts on the network capacity, and the proposed topology control scheme can substantially improve the network capacity in MANETs with cooperative communications.
\end{abstract}

Keywords: COCO topology control, MANETs, Cooperative communications

\section{INTRODUCTION}

\section{A. Background}

In this considering both upper layer network capacity and physical layer cooperative communications, the topology control issues in MANETs with cooperative communications. We propose a Capacity-Optimized Cooperative (COCO) topology control scheme to improve the network capacity in MANETs by jointly optimizing transmission mode selection, relay node selection, and interference control in MANETs with cooperative communications. Through simulations, we show that physical layer cooperative communications have significant impacts on the network capacity, and the proposed topology control scheme can substantially improve the network capacity in MANETs with cooperative communications. The remainder of the article is structured as follows. We introduce cooperative communications and the topology control problem in MANETs. Network capacity and the proposed COCO topology control scheme are presented.

\section{IMPROVING NETWORK CAPACITY USING TOPOLOGY CONTROL IN MANETS WITH COOPERATIVE COMMUNICATIONS}

To improve the network capacity in MANETs with cooperative communications using topology control, we can set the network capacity as the objective function in the topology control problem. In order to derive the network capacity in a MANET with cooperative communications, we need to obtain the link capacity and inference model when a specific transmission manner (i.e., direct transmission, multi-hop transmission, or cooperative transmission) is used.

When traditional direct transmission is used, given a small outage probability, the outage link capacity can be easily derived. Since only two nodes are involved in the direct transmission, the interference set of a direct transmission is the union of coverage sets of the source node and the destination node.

In this paper, we adopt the interference model in, which confines concurrent transmissions in the vicinity of the transmitter and receiver. This model fits the medium access control function well, e.g., the popular IEEE 802.11 MAC in most mobile devices in MANETs. Here in, interference of a link is defined as some combination of coverage of nodes involved in the transmission.

Multi-hop transmission can be illustrated using two-hop transmission. When two-hop transmission is used, two time slots are consumed. In the first slot, messages are transmitted from the source to the relay, and the messages will be forwarded to the destination in the second slot. The outage capacity of this two-hop transmission can be derived considering the outage of each hop transmission. The transmission of each hop has its own interference, which happens in different slots. Since the transmissions of the two hops cannot occur simultaneously but in two separate time slots, the end-to-end interference set of the multi-hop link is determined by the maximum of the two interference sets.

When cooperative transmission is used, a best relay needs to be selected proactively before transmission. In this study, we adopt the decode-and-forward relaying scheme. The source broadcasts its messages to the relay and destination in the first slot. The relay node decodes and reencodes the signal from the source, and then forwards it to the destination in the second slot. The two signals of the source and the relay are decoded by maximal rate combining at the destination. The maximum instantaneous end-to-end mutual information, outage probability, and outage capacity can be derived. For the interference model, in the broadcast period, both the covered neighbors of the source and the covered neighbors of the relay and the destination have to be silent to ensure successful receptions. In the second slot, both the covered neighbors of the selected relay and the destination have to be silent to ensure successful receptions. 
After obtaining the link capacity and inference models, the network capacity can be derived as the objective function in the topology control problem. By considering direct transmission, multi-hop transmission, cooperative transmission, and interference, the proposed $\mathrm{COCO}$ topology control scheme extends physical layer cooperative communications from the link-level perspective to the network-level perspective in MANETs. The proposed scheme can determine the best type of transmission and the best relay to optimize network capacity.

Two constraint conditions need to be taken into consideration in the proposed $\mathrm{COCO}$ topology control scheme. One is network connectivity, which is the basic requirement in topology control. The end-to-end network connectivity is guaranteed via a hop-by-hop manner in the objective function. Every node is in charge of the connections to all its neighbors. If all the neighbor connections are guaranteed, the end-to-end connectivity in the whole network can be preserved. The other aspect that determines network capacity is the path length. An end-toend transmission that traverses more hops will import more data packets into the network. Although path length is mainly determined by routing, COCO limits dividing a long link into too many hops locally. The limitation is two hops due to the fact that only two-hop relaying is adopted.

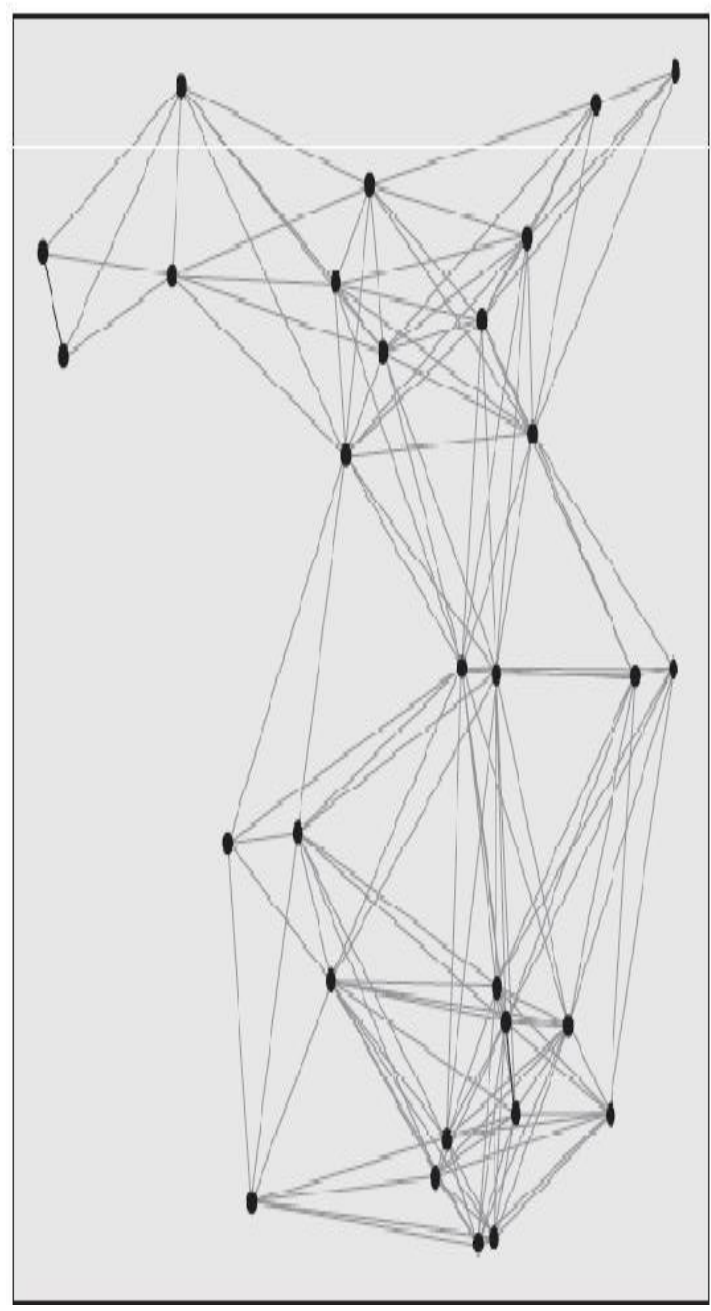

Fig.1 Different nodes in MANET

\section{RESULTS}

Evaluation of results:

In this section, the performance of the proposed scheme is illustrated using computer simulations. We consider a MANET with 30 nodes randomly deployed in a $800 \times 800$ $\mathrm{m} 2$ area. The number of nodes is changed in the simulations. The channels follow a Raleigh distribution. We compare the performance of the proposed scheme with that of an existing well-known topology control scheme, called LLISE, which only considers traditional multi-hop transmissions without cooperative communications and preserves the minimum interference path for each neighbor link locally. We also show the worst network capacity among all the topology configurations for comparison. The original topology where links exist whenever the associated two end nodes are within transmission range of each other. It is clear that this topology lacks any physical layer cooperative communications. The resulting topology using the proposed COCO topology control scheme. The solid lines denote traditional direct transmissions and multi-hop transmissions, and the dash lines denote links involved in cooperative communications. To maximize the network capacity of the MANET, many links in the network are involved in cooperative communications. The network capacity per node in different topology control schemes with different numbers of nodes in the MANET. The proposed COCO scheme has the highest network capacity regardless of the number of nodes in the network. Similar to COCO, LLISE is executed in each node distributedly. It preserves all the edges on the minimum interference path for each link in the resulting topology, thus minimizes the interference to improve network capacity. Nevertheless, COCO can achieve a much higher network capacity than LLISE, since LLISE only considers Multihop transmissions. The performance gain of the proposed scheme comes from the joint design of transmission mode selection, relay node selection, and interference minimization in MANETs with cooperative communications.

\section{CONCLUSIONS}

In this paper introduced physical layer cooperative communications, topology control, and network capacity in MANETs. To improve the network capacity of MANETs with cooperative communications, and also proposed a Capacity- Optimized Cooperative (COCO) topology control scheme that considers both upper layer network capacity and physical layer relay selection in cooperative communications. Simulation results have shown that physical layer cooperative communications techniques have significant impacts on the network capacity, and the proposed topology control scheme can substantially improve the network capacity in MANETs with cooperative communications. Future work is in progress to consider dynamic traffic patterns in the proposed scheme to further improve the performance of MANETs with cooperative communications. 


\section{REFERENCES}

[1] J. Laneman, D. Tse, and G. Wornell, "Cooperative diversity in wireless networks: Efficient protocols and outage behavior," IEEE Trans. Inform. Theory, vol. 50, no. 12, pp. 3062-3080, 2004.

[2] V. Mahinthan, L. Cai, J. Mark, and X. Shen, "Partner selection based on optimal power allocation in cooperative-diversity systems," IEEE Trans. Veh. Tech., vol. 57, pp. 511 -520, Jan. 2008.

[3] P. H. J. Chong, F. Adachi, S. Hamalainen, and V. Leung, "Technologies in multihop cellular network," IEEE Commun. Magazine, vol. 45, pp. 64-65, Sept. 2007.

[4] K. Woradit, T. Quek, W. Suwansantisuk, M. Win, L. Wuttisittikulkij, and $\mathrm{H}$. Wymeersch, "Outage behavior of selective relaying schemes," IEEE Trans. Wireless Commun., vol. 8, no. 8, pp. 3890-3895, 2009.

[5] P. Santi, "Topology control in wireless ad hoc and sensor networks," ACM Computing Surveys (CSUR), vol. 37, no. 2, pp. 164-194, 2005.

[6] T. Cover and A. E. Gamal, "Capacity theorems for the relay channel," IEEE Trans. Inform. Theory, vol. 25, pp. 572-584, Sept. 1979.14

[7] Q. Guan, S. Jiang, Q. Ding, and G. Wei, "Impact of topology control on capacity of wireless ad hoc networks," in Proc. IEEE ICCS, (Guangzhou, P.R. China), Nov. 2008.

[8] P. Gupta and P. Kumar, "The capacity of wireless networks," IEEE Trans. Inform. Theory, vol. 46, no. 2, pp. 388-404, 2000.

9] A. Goldsmith, Wireless Communications. Cambridge University Press, 2005.

[10] M. Burkhart, P. von Rickenbach, R. Wattenhofer, and A. Zollinger, "Does topology control reduce interference?," in Proc. 5th ACM Int. Symposium on Mobile Ad Hoc Networking and Computing, (Tokyo, Japan), pp. 9-19, May 2004.

[11] Y. Wei, F. R. Yu, and M. Song, "Distributed Optimal Relay Selection in Wireless Cooperative Networks with Finite-State Markov Channels," IEEE Trans. Vehic. Tech., vol. 59, June 2010, pp. $2149-58$.

[12] Yan Gao , Hou, J.C. , Hoang Nguyen, “Topology Control for Maintaining Network Connectivity and Maximizing Network Capacity Under the Physical Model" INFOCOM 2008 IEEE April 2008 pp:1013 - 1021.

[13] D. Djenouri, L. Khelladi, and N. Badache, "A survey of security issues in mobile ad hoc and sensor networks," IEEE Commun. Surveys Tutorials, vol. 7, pp. 2-28, 2005.

[14] P. Michiardi and R. Molva, "Simulation-based analysis of security exposures in mobile ad hoc networks," in Proc. European Wireless 2002: Next Generation Wireless Networks: Technologies, Protocols, Services Applications, 2002.

[15] L. Blazevic, L. Buttyan, S. Capkun, S. Giordano, J.-P. Hubaux, and J.-Y. L. Boudec, "Self-organization in mobile ad hoc networks: The approach of terminodes," IEEE Commun. Mag., vol. 39, pp. 166-174, 2001.

[16] S. Kent and R. Atkinson, "Security Architecture for the Internet Protocol," IETF RFC 2401, Nov. 1998.

[17] C.-S. Hsu, J.-P. Sheu, and S.-C. Tung, "An On-Demand Bandwidth Reservation QoS Routing Protocol for Mobile Ad Hoc Networks,"

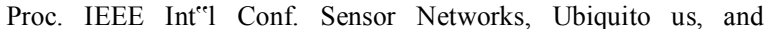
Trustworthy Computing, vol. 2, pp. 198-207, 2006. 